\title{
Correction to: Bounded Isometries and Homogeneous Quotients
}

\author{
Joseph A. Wolf ${ }^{1}$
}

Published online: 9 December 2021

() Mathematica Josephina, Inc. 2021

\section{Correction to: The Journal of Geometric Analysis (2017) 27:56-64 https://doi.org/10.1007/s12220-015-9672-9}

A few years ago Profs. Yurii Nikonorov and Ming Xu found a gap [1] in my paper [2] on homogeneity and bounded isometries. In lines 2 and 3 of the third paragraph of the proof of [2, Theorem 2.5] it was only shown that $A d(s k)$ is bounded, and from that it should have been shown that $A d(s)$ is bounded. Now Prof. Nikonorov modified my original proof, completing the argument, and agreed to its publication as a correction. See below. The statement of the theorem is unchanged; the modified argument uses a result [3] of Moskowitz.

Theorem ([2, Theorem 2.5]). Let $(M, d)$ be a metric space on which an exponential solvable Lie group $S$ acts effectively and transitively by isometries. Let $G=I(M, d)$. Then $G$ is a Lie group, any isotropy subgroup $K$ is compact, and $G=S K$. If $g \in G$ is a bounded isometry then $g$ is a central element in $S$.

Proof (Yurii Nikonorov [4]). $M$ carries a differentiable manifold structure for which $s \mapsto s\left(x_{0}\right)$ is a diffeomorphism $S \cong M$. In the compact-open topology $G=I(M, d)$ is locally compact and its its action on $M$ is proper [5]. In particular, if $x_{0} \in M$ then the isotropy subgroup $K=\left\{k \in G \mid k\left(x_{0}\right)=x_{0}\right\}$ is compact. Further [6, Corollary in $\S 6.3] G$ is a Lie group. Now $S$ and $K$ are closed subgroups, $G=S K$, and $M=G / K$. The action of $G$ on $M=S K / K=S$ is $(s k): s^{\prime} \mapsto s \cdot k s^{\prime} k^{-1}$. We now assume that $x_{0}=1 \in S$.

Express $g=s k \in G$ with $s \in S$ and $k \in K$. Suppose that $g$ is a bounded isometry of $(M, d)$. So there is a compact set $C \subset S$ such that $\tau(g)\left(s^{\prime}\right) s^{\prime-1}=s\left[\left(k s^{\prime} k^{-1}\right) s^{\prime-1}\right] \in C$ for every $s^{\prime} \in S$. Then $\left(k s^{\prime} k^{-1}\right) s^{\prime-1} \in s^{-1} C$ so the automorphism $a: S \rightarrow S$, $a\left(s^{\prime}\right)=k s^{\prime} k^{-1}$ is an automorphism of bounded displacement. By Corollary 1.3 in [3],

The original article can be found online at https://doi.org/10.1007/s12220-015-9672-9.

Joseph A. Wolf

jawolf@math.berkeley.edu

1 Department of Mathematics, University of California, Berkeley, CA 94720-3840, USA 
we see that $a$ is trivial. Since the isotropy representation is faithful, it follows that $k=1$ and $g=s \in S$.

Express $g=s=\exp (\xi)$ where $\xi \in \mathfrak{s}:=\operatorname{Lie}(S)$. Decompose $\mathfrak{s}$ as a vector space direct sum $\mathfrak{n}+\mathfrak{a}$, where $\mathfrak{n}$ is the nilradical of $\mathfrak{s}$. In a basis respecting that direct sum, $\left.\operatorname{ad}(\xi)\right|_{\mathfrak{s}}$ has matrix of the form $\left(\begin{array}{ll}0 & h \\ 0 & 0\end{array}\right)$. Let $N$ denote the unipotent radical of $S$, so $\mathfrak{n}:=\operatorname{Lie}(N)$. Then the $(1,1)$ and $(2,1)$ blocks vanish because $g$ centralizes $N$ by $[7$, Théorème 1], and the $(2,2)$ block vanishes because $[\mathfrak{s}, \mathfrak{s}] \subset \mathfrak{n}$. Note $\left(\begin{array}{ll}0 & h \\ 0 & 0\end{array}\right)^{2}=0$, so $\left.\operatorname{Ad}(g)\right|_{\mathfrak{s}}-I=\left.\operatorname{ad}(\xi)\right|_{\mathfrak{s}}$. It cannot have relatively compact image unless $h=0$. Thus $\left.\operatorname{Ad}(g)\right|_{\mathfrak{s}}=I$, and $g \in\left(Z_{G}(S) \cap S\right)=Z_{S}$ as asserted.

\section{References}

1. Nikonorov, Y., Xu, M.: Private communications (2019)

2. Wolf, J.A.: Bounded isometries and homogeneous quotients. J. Geom. Anal. 27, 56-64 (2017)

3. Moskowitz, M.: Some remarks on automorphisms of bounded displacement and bounded cocycles. Monatsh. Math. 85, 323-336 (1978)

4. Nikonorov, Y.: Private communication (2021)

5. van Dantzig, D., van der Waerden, B.L.: Über metrisch homogene Räume. Abh. Math. Sem. Hamburg 6, 367-376 (1928)

6. Montgomery, D., Zippin, L.: Topological Transformation Groups. Interscience, New York (1955)

7. Tits, J.: Automorphismes à deplacement borne des groupes de Lie. Topology 3, 97-107 (1964)

Publisher's Note Springer Nature remains neutral with regard to jurisdictional claims in published maps and institutional affiliations. 